\title{
Reproducibility of mRNA-Based Testing of ESR1, PGR, ERBB2, and MKI67 Expression in Invasive Breast Cancer-A Europe-Wide External Quality Assessment
}

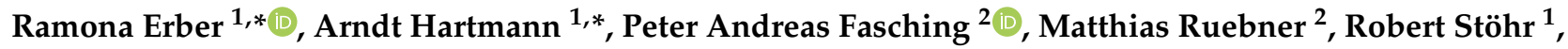 \\ Matthias Wilhelm Beckmann ${ }^{2}$, Miriam Zentgraf ${ }^{1}$, Verena Popp ${ }^{1}$, Jodi Weidler ${ }^{3}$, Iris Simon ${ }^{3}$, Steffi Becker ${ }^{3}$, \\ Hanna Huebner ${ }^{2}{ }^{-}$, Josephine Fischer ${ }^{4}$, Elena Guerini Rocco ${ }^{5,6}$, Giuseppe Viale ${ }^{5,6}$, Anne Cayre ${ }^{7}$, \\ Frederique Penault-Llorca ${ }^{7}$, Tamara Caniego Casas ${ }^{8,9}$, Belén Pérez-Miés $8,9,10,11$, José Palacios $8,9,10,11 \oplus$, \\ Paul Jank ${ }^{12} \oplus$, Carsten Denkert ${ }^{12}$, Lina Khoury ${ }^{13}$, Thomas Mairinger ${ }^{13}$ and Fulvia Ferrazzi ${ }^{1,14} \oplus$
}

1 Institute of Pathology, University Hospital Erlangen, Friedrich-Alexander-Universität Erlangen-Nürnberg (FAU), Comprehensive Cancer Center Erlangen-EMN (CCC ER-EMN), 91054 Erlangen, Germany; robert.stoehr@uk-erlangen.de (R.S.); miriam.zentgraf@gmx.net (M.Z.); verena.popp@uk-erlangen.de (V.P.); fulvia.ferrazzi@uk-erlangen.de (F.F.)

2 Department of Gynecology and Obstetrics, University Hospital Erlangen, Comprehensive Cancer Center Erlangen-EMN (CCC ER-EMN), Friedrich-Alexander-Universität Erlangen-Nürnberg (FAU), 91054 Erlangen, Germany; peter.fasching@uk-erlangen.de (P.A.F.); matthias.ruebner@uk-erlangen.de (M.R.); matthias.beckmann@uk-erlangen.de (M.W.B.); hanna.huebner@uk-erlangen.de (H.H.)

check for

updates

Citation: Erber, R.; Hartmann, A.; Fasching, P.A.; Ruebner, M.; Stöhr, R.; Beckmann, M.W.; Zentgraf, M.; Popp,

V.; Weidler, J.; Simon, I.; et al.

Reproducibility of mRNA-Based

Testing of ESR1, PGR, ERBB2, and $M K I 67$ Expression in Invasive Breast Cancer-A Europe-Wide External Quality Assessment. Cancers 2021, 13 4718. https://doi.org/10.3390/ cancers 13184718

Academic Editor: David Wong

Received: 10 August 2021

Accepted: 8 September 2021

Published: 21 September 2021

Publisher's Note: MDPI stays neutral with regard to jurisdictional claims in published maps and institutional affiliations.

Copyright: (C) 2021 by the authors Licensee MDPI, Basel, Switzerland. This article is an open access article distributed under the terms and conditions of the Creative Commons Attribution (CC BY) license (https:// creativecommons.org/licenses/by/ $4.0 /)$
3 Cepheid, Department of Medical and Scientific Affairs and Strategy, Oncology, 904 Caribbean Drive, Sunnyvale, CA 94089, USA; Jodi.Weidler@cepheid.com (J.W.); irissimon@yahoo.com (I.S.); Steffi.Becker@cepheid.com (S.B.)

4 Qualitätssicherungs-Initiative Pathologie QuIP GmbH, 10117 Berlin, Germany; Fischer@quip.eu

5 Department of Pathology and Laboratory Medicine, IEO, European Institute of Oncology, IRCCS, 20141 Milan, Italy; Elena.GueriniRocco@ieo.it (E.G.R.); giuseppe.viale@ieo.it (G.V.)

6 Department of Oncology and Hemato-Oncology, University of Milan, 20122 Milan, Italy

7 Centre Jean Perrin, Department of Pathology and Clermont Auvergne University, INSERM, U1240, "Imagerie Moléculaire et Stratégies Théranostiques", F-63011 Clermont Ferrand, France; Anne.CAYRE@clermont.unicancer.fr (A.C.); Frederique.PENAULT-LLORCA@clermont.unicancer.fr (F.P.-L.)

8 Instituto Ramón y Cajal de Investigación Sanitaria, 28034 Madrid, Spain; tamara880723@hotmail.com (T.C.C.) bperezmies@gmail.com (B.P.-M.); jose.palacios@salud.madrid.org (J.P.)

9 CIBER-ONC, Instituto de Salud Carlos III, 28029 Madrid, Spain

10 Department of Pathology, Hospital Universitario Ramón y Cajal, 28034 Madrid, Spain

11 Facultad de Medicina, Universidad de Alcalá de Henares, 28871 Madrid, Spain

12 Institute of Pathology, UKGM-University Hospital Marburg, Philipps-University Marburg, 35043 Marburg, Germany; paul.jank@uni-marburg.de (P.J.); carsten.denkert@uni-marburg.de (C.D.)

13 MVZ Helios Hospital Emil von Behring GmbH, 14165 Berlin, Germany; lina.khoury@helios-gesundheit.de (L.K.); thomas.mairinger@helios-gesundheit.de (T.M.)

14 Department of Nephropathology, Institute of Pathology, Friedrich-Alexander-Universität Erlangen-Nürnberg (FAU), 91054 Erlangen, Germany

* Correspondence: ramona.erber@uk-erlangen.de (R.E.); arndt.hartmann@uk-erlangen.de (A.H.); Tel.: +49-9131-85-43634 (R.E.); +49-9131-85-22287 (A.H.)

Simple Summary: Four biomarkers [estrogen receptor (ER), progesterone receptor (PgR), Ki-67, and HER2], are used to stratify breast cancer (BC) into subtypes predictive of therapy response. In a Europe-wide external quality assessment, we compared performance of an mRNA-based method [Xpert ${ }^{\circledR}$ Breast Cancer STRAT4 (CE-IVD)] for determining ESR1, PGR, ERBB2, and MKI67 expression against the gold standard [immunohistochemistry (IHC)/HER2 in situ hybridization (ISH)]. The coordinating center (CC) and five European laboratories tested ten breast cancer samples. STRAT4 binary (positive or negative) results of each marker were compared with the gold standard. ESR1 and ERBB2 mRNA results were concordant with IHC/ISH in all single analyses. In contrast, PGR and MKI67 results were discordant in a few cases, which had STRAT4 expression values close to assay cut-offs and immunohistochemically presented heterogeneous low positive PgR and heterogeneous Ki-67. STRAT4 assay may be a reproducible method. However, cases with expression values close to cut-offs should be carefully reviewed. 


\begin{abstract}
Estrogen receptor (ER), progesterone receptor (PgR), Ki-67, and HER2 immunohistochemistry (IHC) together with HER2 in situ hybridization (ISH) are utilized to classify invasive breast cancer (IBC) into predictive molecular subtypes. As IHC evaluation may be hampered by analytical errors, gene expression assays could offer a reliable alternative. In this first Europe-wide external quality assessment (EQA) study, we investigated performance of mRNA-based Xpert ${ }^{\circledR}$ Breast Cancer STRAT4 (CE-IVD) in five European laboratories. The cohort comprised ten pre-therapy IBC core biopsies diagnosed in the coordinating center (CC). STRAT4 binary (positive or negative) mRNA results of each marker (ESR1, PGR, ERBB2, MKI67) were compared with the gold standard IHC/ISH performed by the CC. Sensitivity, specificity, and accuracy of ESR1 and ERBB2 mRNA were $100 \%$ for all samples. In contrast, PGR expression was falsely negative for one case by two sites and MKI67 falsely negative for two cases (respectively by four and one sites). These cases had STRAT4 expression values close to assay cut-offs and immunohistochemically presented heterogeneous low positive PgR and heterogeneous Ki-67. Our EQA shows that STRAT4 mRNA assay may be a reproducible method to evaluate ER, PgR, HER2, and Ki-67 status. However, cases with expression values close to assay cut-offs should be carefully reviewed.
\end{abstract}

Keywords: breast cancer; ESR1; PGR; ERBB2; MKI67; mRNA expression testing; STRAT4; GeneXpert; Xpert

\title{
1. Introduction
}

Invasive breast cancer (IBC) can be classified into different gene expression-based subtypes, namely Luminal A, Luminal B, HER2-enriched, and basal-like, which are associated with different prognosis and different response to therapy [1-3]. In clinical routine, the immunohistochemical (IHC) determination of estrogen receptor (ER, gene/mRNA ESR1), progesterone receptor (PgR, gene/mRNA PGR), human epidermal growth factor receptor 2 (HER2, gene synonym: ERBB2) and Ki-67 (gene/mRNA MKI67) are used to approximate these molecular subtypes. Using these markers, IBC can be classified into molecular-like breast cancer subtypes: hormone receptor-positive tumors, which include Luminal Alike and Luminal B-like (either HER2-negative or HER2-positive), HER2-positive tumors (HER2+, non-luminal), and triple-negative breast cancer (TNBC) [4,5].

The IHC assessment of ER, PgR, HER2, and Ki-67 requires standardized staining and evaluation procedures. However, IHC protocols for each of these four biomarkers are linked to some difficulties: IHC staining may be hampered by many pre-analytical and analytical errors, e.g., inadequate fixatives / fixation, decalcification, age of archived unstained slides, sensitivity of antibody clones, and detection kits. In addition, discussions on the optimal IHC cut-offs for biomarker positivity are still on-going. Indeed, cut-offs that are clinically of prognostic and/or predictive value have to be assessed performing comprehensive clinical trials [6,7]. For ER/PgR, HER2, and Ki-67, cut-off recommendations have changed several times in the last 20 years [5,6,8-19]. Moreover, in the case of ER IHC, there is controversy on the current cut-off of $\geq 1 \%$, since some studies have shown that IBCs with low ER expression (1-9\% or 1-10\%; cut-offs depending on different guidelines/references) behave more like ER-negative tumors [9,20-22]. Difficulties also exist for HER2, as in cases of HER2 IHC score equal to 2+ (borderline/equivocal), IHC has to be complemented by in situ hybridization (ISH), which is another very sensitive assay that requires standardized reading and optimal cut-offs [12]. Regarding Ki-67, a standardized protocol and cut-off are still lacking $[6,15]$, although efforts to harmonize Ki-67 assessment have been published recently [23].

Due to the aforementioned issues, the pathological assessment of molecular-like breast cancer subtypes using IHC/ISH might be challenging, especially in low- and middleincome countries (LMIC). The use of a standardized, robust, reliable, and cost-effective mRNA-based test for the four biomarkers, ER (ESR1), PgR (PGR), HER2 (ERBB2), and Ki-67 (MKI67), may offer a good alternative to IHC. Some assays have already been 
validated and are commercially available $[24,25]$. Among these, the validity, robustness, and reproducibility of the Xpert ${ }^{\circledR}$ Breast Cancer STRAT4 test (CE-IVD, in vitro diagnostic medical device. Not available in the U.S. Not available in all countries.) ("STRAT4") (Cepheid, Sunnyvale, CA, USA) have been shown in large breast cancer cohorts [25-27]. Indeed, one blinded, retrospective study reported high concordance between biomarker expression detection by STRAT4 and IHC with receiver operating characteristic curve (ROC) area under the curve (AUC) values of $0.99,0.95,0.99$, and 0.85 for ESR1, PGR, ERBB2, and $M K I 67$, respectively [26]. However, in most European countries, regular participation in Europe-wide external quality assessment (EQA) programs is required for pathology labs and tumor centers to receive laboratory accreditation. Until now, there have been EQA studies for ER/PgR/HER2/Ki-67 by IHC and HER2 ISH testing, but not for mRNA-based assessment of ESR1/PGR/ERBB2/MKI67. Here, we present the first Europe-wide EQA study for one cartridge-based "four-marker" gene expression assay, the STRAT4 assay, evaluating its reproducibility and correlation to the gold standard (IHC/ISH) in different pathologic laboratories across Europe.

\section{Materials and Methods}

\subsection{Sample Cohort}

Ten cases of diagnostic pre-therapy breast core biopsies with invasive breast cancer (IBC) that had been diagnosed in the coordinating center (CC) between 2016 and 2018 were included in the EQA study. These cases contained six ER+/PgR+ IBC with varying proliferation rates, (Ki67), two HER2+, and two TNBC (Table 1). Each sample had to feature sufficient formalin-fixed and paraffin-embedded tissue to guarantee that sufficient diagnostic material would be left after the EQA study in case of necessary re-evaluation after tumor progression. Tumor cellularity had to be $\geq 30 \%$. Moreover, cohort cases were not allowed to be included in any clinical trial. Pathological features, including histological tumor type, as well as IHC and ISH data for ER, PgR, HER2, and Ki-67 were extracted from the original pathology report. For each case, Xpert $^{\circledR}$ Breast Cancer STRAT4 (ESR1, PGR, ERBB2, and MKI67) mRNA test was performed in the CC and STRAT4 results for each marker had to match IHC/ISH subtyping. The Ethics Committee of the Medical Faculty of Erlangen University Hospital approved the study [ref. numbers 2700 (approval date: 1 March 2013) and 297_17 Bc (approval date: 11 January 2018)], and all patients provided written informed consent.

\subsection{Immunohistochemical (IHC) Expression of ER, PgR, HER2, and Ki-67 and IHC Subtyping}

IHC was conducted on FFPE IBC tissue of the preoperative core biopsies according to the established and accredited routine standards of the CC and manufacturer's instruction manual with an automated staining module (BenchMark ULTRA IHC/ISH staining module, Ventana Medical Systems, Oro Valley, AZ, USA). For assessment of ER, PgR, and Ki-67 IHC status, a monoclonal rabbit antibody against ER-alpha (clone EP1, 1:40 dilution, DAKO, Glostrup, Denmark), a monoclonal mouse antibody against PgR (clone pgR636, 1:200 dilution, DAKO, Glostrup, Denmark), and a monoclonal antibody against Ki-67 (clone MIB-1, 1:100 dilution, DAKO, Glostrup, Denmark) were used. The continuous percentage of positively stained tumor cells was stated in the pathology reports. Moreover, staining intensity and the immunoreactive score (IRS) according to Remmele and Stegner were reported for ER and PgR [28]. For HER2 IHC staining, a polyclonal antibody against HER2 (1:200 dilution, DAKO, Glostrup, Denmark) was used, and HER2 IHC score was documented in the pathology reports as $0,1+, 2+$, or 3+ in accordance with the published recommendations [11]. Tumors with a score of 0 or $1+$ were regarded as HER2-negative, and cases with a score of 3+ were considered as HER2 positive. Breast cancer samples with a $2+$ staining were analyzed for gene copy number (GCN) alteration of HER2 using chromogenic in situ hybridization (CISH). The HER2 GCN and the centromere of the corresponding chromosome 17 were visualized using a kit with two probes of different colors (ZytoDot ${ }^{\circledR}$ 2C SPEC ERBB2/CEN17 Probe, ZytoVision GmbH, Bremerhaven, Germany). A case was defined as HER2 amplified/positive if the HER2/CEN17 ratio was $\geq 2.0$ or if the HER2 GCN was $\geq 6.0$ signals per tumor nuclei [11]. 


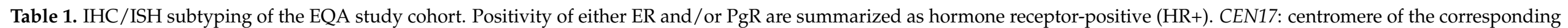
chromosome 17; n.p.: not performed.

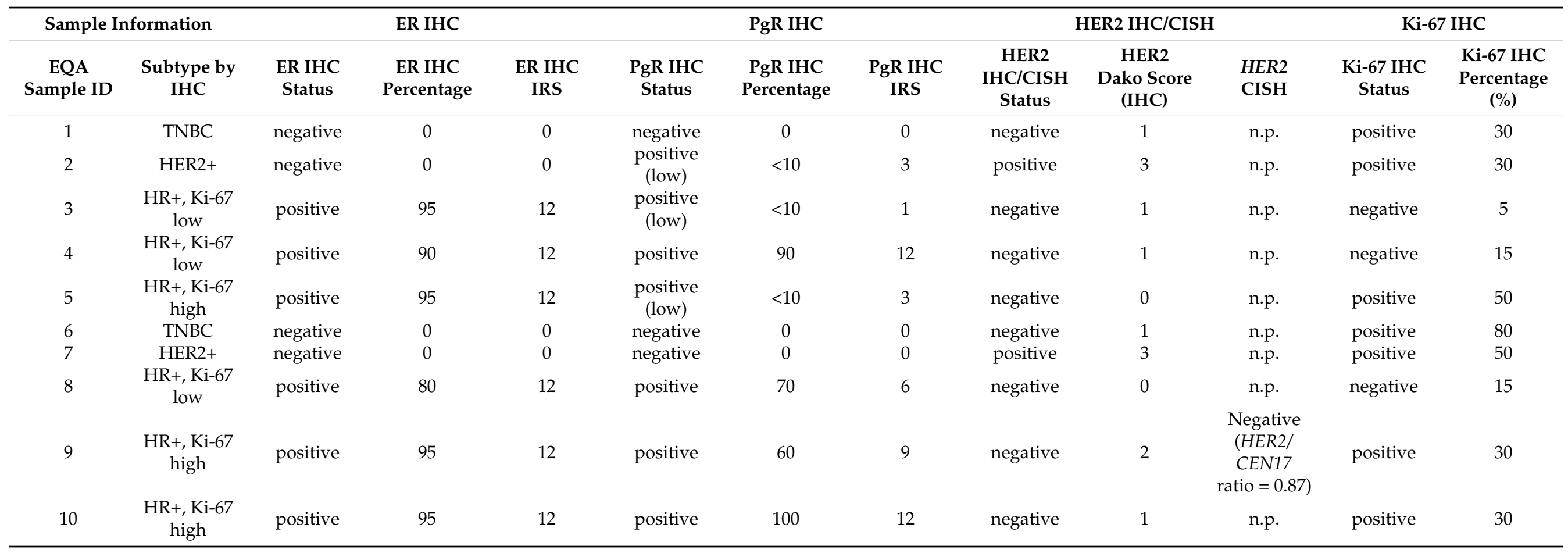


IHC (and for HER2, IHC/CISH) binary (positive/negative) status was defined as follows:

a. Samples with ER or PgR IHC $\geq 1 \%$ positive stained cells, regardless of staining intensity, were classified as positive ( $<10 \%$ as low positive); samples with $<1 \%$ cells staining at any intensity were classified as negative $[8,9,20,21]$. ER and/or PgR positivity were summarized as hormone receptor positivity $(\mathrm{HR}+)$.

b. Samples with HER2 IHC 0 or $1+$ were classified as negative, HER2 IHC 3+ were defined as positive; cases with IHC $2+$ results required reflex testing by CISH to determine positive or negative results [11,12,29].

c. Samples with $\mathrm{Ki}-67>20 \%$ cells staining positive, regardless of staining intensity, were classified as high/positive. Samples with $\leq 20 \%$ cells staining at any intensity were counted as low/negative [5,14,30].

IHC/ISH breast cancer subtyping was concluded as follows: Cases that were negative for ER, PgR, and HER2 were regarded as TNBC, those with HER2 positivity as HER2+ subgroup, and IBC with ER and/or PgR positivity but HER2 negativity as HR+ subtype. This group was further divided into $\mathrm{HR}+$ with low versus high $\mathrm{Ki}-67$ expression. IHC status of each marker (ER, PgR, HER2, and Ki-67) and IHC/ISH subtyping of the ten EQA IBC samples are reported in Table 1.

\section{3. $m R N A$-Based Testing of ESR1, PGR, ERBB2, and MKI67 in the Coordinating Center}

The Xpert ${ }^{\circledR}$ Breast Cancer STRAT4 (CE-IVD*) mRNA assay (Cepheid, Sunnyvale, CA, USA) is a Real Time-quantitative Polymerase Chain Reaction (RT-qPCR) based semiquantitative assay to analyze ESR1, PGR, ERBB2, and MKI67 mRNA levels isolated from FFPE IBC tissue. CC performed the test according to the manufacturer's instructions. Archived FFPE blocks of the ten cases were used as starting material. For each block, one hematoxylin and eosin (H\&E) stained slide containing a single $2 \mu \mathrm{m}$ stained section for confirmation of breast cancer diagnosis and tumor area as well as one unstained slide containing a single $10 \mu \mathrm{m}$ tissue section intended for mRNA testing were created. After reviewing the IBC and marking the tumor area on the H\&E slide by a pathologist experienced in breast cancer, the FFPE tumor area was removed (scraped using a single use razor blade) from the unstained slide and then was prepared into a tissue lysate using the FFPE lysis kit (Cepheid, Sunnyvale, CA, USA) following the manufacturer's instructions per the STRAT4 product insert. A $520 \mu \mathrm{L}$ aliquot of the prepared tissue specimen lysate was then transferred to the sample chamber of a STRAT4 cartridge and put into a GeneXpert ${ }^{\circledR}$ Instrument module for automated RNA extraction, purification, and RTqPCR analysis [Cepheid, Sunnyvale, CA, USA, (http:/ / www.cepheid.com/us/cepheidsolutions/systems/genexpert-systems/genexpert-i, accessed on 29 March 2020)]. The detailed protocol as well as the GeneXpert ${ }^{\circledR}$ DX software analysis settings of the automated diagnostic platform have been previously described [26]. The assay uses cytoplasmic FMR1-interacting protein 1 (CYFIP1) as the sample adequacy control and reference gene to normalize the mRNA expression levels. In addition to the binary results and cycle threshold $(\mathrm{Ct})$ values of the internal reference target CYFIP1, the software outputs delta cycle threshold $(\mathrm{dCt})$ values, as well as binary test results, of each biomarker of interest (ESR1, PGR, ERBB2, and MKI67). The dCt value is defined as difference between the CYFIP1 $C t$ and the $C t$ of the target gene $\left(C_{C Y F I P 1}\right)-\left(C t_{\text {target gene }}\right)$ [26]. The $\mathrm{dCt}$ value is then compared to a prespecified validated cut-off to define the binary (positive/negative) marker result for each target analyte.

\subsection{EQA Implementation}

Five European (France, Germany, Italy, Spain) pathology laboratories (here de-identified as EQA sites A-E) participated in the EQA study. All participants had already established the diagnostic platform and corresponding GeneXpert Dx software (including assay definition files) for STRAT4 testing in their laboratories and participated in a training for the Xpert $^{\circledR}$ Breast Cancer STRAT4 mRNA analysis (as was the case for the CC). Each 
site received one $2 \mu \mathrm{m}$ thick H\&E stained slide and one $10 \mu \mathrm{m}$ thick adjacent unstained slide, prepared and shipped by the CC, for each of the 10 patient de-identified clinical test specimens for STRAT4 analysis. Xpert ${ }^{\circledR}$ Breast Cancer STRAT4 kits and FFPE lysis reagents were directly provided by Cepheid. Each laboratory performed the mRNA assay according to the aforementioned criteria/protocol. Raw data of the test result were then transferred by participating laboratories directly to the CC for statistical analysis.

\subsection{Re-Evaluation of Discrepant Cases}

After the EQA analysis, for the cases that received discrepant results by the participating sites with respect to the CC, the CC H\&E slide, as well as initial CC IHC stainings of the discrepant marker(s), were reviewed. Moreover, deeper sections (following the sections created for STRAT4 analysis at the five EQA sites) were cut, and IHC as well as STRAT4 analysis were repeated and compared with the initial CC results.

\subsection{Statistical Analysis}

Test results received from the participating centers were collected by the CC. In addition to the pathological parameters (tumor grading, IHC) of each EQA sample, binary mRNA test results as well as $\mathrm{dCt}$ values of the internal reference gene and each biomarker of interest (ESR1, PGR, ERBB2 and MKi67), reported from each site, were recorded.

Data analysis was performed in the R statistical environment v. 3.6.1 (R Core Team, 2019) [31]. Sensitivity, specificity, precision (positive predictive value), and accuracy for each marker and each of the five participating centers were calculated by comparing the binary test results with the IHC status assessed by the CC, taken as the gold standard. Confidence intervals for the average performance indicators across the five centers were calculated relying on a $t$ distribution.

\section{Results}

\subsection{Comparison of Binary STRAT4 Results}

The primary objective of our study was to compare the binary results of each marker, separately for each center, with the binary (positive/negative) IHC status (and additionally HER2 CISH, if necessary) assessed by the CC, considered as the gold standard. Each site passed the technical quality criteria of the assay and received evaluable results from STRAT4 testing for each of the ten EQA samples and each marker. Overall, all centers passed the EQA study criteria, which required an agreement of at least $80 \%$ between test results for each marker and the CC IHC. The average sensitivity, specificity, and accuracy (overall concordance) of ESR1 and ERBB2 mRNA testing across all five participating sites were $100 \%$ for all samples. Instead, PGR mRNA had a discordant negative result for one case (case \#2) by two sites, and thus had an average sensitivity of $94 \%$, specificity of $100 \%$, and accuracy of $96 \%$. MKI67 mRNA was discordant negative for two cases (case \#2 by four sites, $\# 10$ by one site), thus having average sensitivity of $86 \%$, specificity of $100 \%$ and accuracy of $90 \%$ (Table 2 ).

The second study end point was to compare the quantitative Xpert ${ }^{\circledR}$ Breast Cancer STRAT4 dCt results for each marker with the percentage staining results by IHC for ER, PgR and Ki-67 as well as HER2 categorical results (negative $=$ IHC 0, 1+, 2+/ISH non-amplified versus positive $=$ IHC $3+$ or IHC $2+/ \mathrm{CISH}$ amplified). However, these analyses were for descriptive purposes and not required for passing the study. Across the coordination center and the EQA sites A-E, the reference gene CYFIP1 cycle threshold (Ct) measurements were between 21-28 for all samples, which was well below the valid Ct cut-off of 35 (Figure 1A). Regarding ESR1 and ERBB2, dCt measurements across all sites for all samples were comparable (Figure 1B,D). Instead, PGR dCt values for the IHC-positive case \#2 were below the STRAT4 dCt cut-off by two sites, thus resulting in discordant negative PGR binary results (Figure 1C). In addition, MKI67 dCt measurements were below the cut-off for two IHC-positive cases. In particular, case \#2 was reported as discrepant mRNA negative by four sites, with the $\mathrm{dCt}$ of one site differing by only 0.1 from the cut-off, and case \#10 
was reported as discordant negative by one site, also with a difference from the cut-off of 0.1 (Figure 1E).

Table 2. Comparison of IHC/ISH status with the STRAT4 binary results. The table shows the average sensitivity, specificity, precision (PPV), and accuracy across all five participating sites (in parenthesis the $95 \%$ confidence interval). PPV: positive predictive value.

\begin{tabular}{ccccc}
\hline $\begin{array}{c}\text { Performance } \\
\text { Index }\end{array}$ & ER & PgR & HER2 & Ki-67 \\
\hline Sensitivity & 1 & 0.94 & 1 & 0.86 \\
& $(1-1)$ & $(0.87-1)$ & $(1-1)$ & $(0.76-0.95)$ \\
Specificity & 1 & 1 & 1 & 1 \\
& $(1-1)$ & $(1-1)$ & $(1-1)$ & $(1-1)$ \\
Precision (PPV) & 1 & 1 & $(1-1)$ & 1 \\
& $(1-1)$ & 0.96 & 1 & $(1-1)$ \\
Accuracy & 1 & $(0.91-1)$ & $(1-1)$ & 0.9 \\
& $(1-1)$ & & & $(0.83-0.97)$ \\
\hline
\end{tabular}
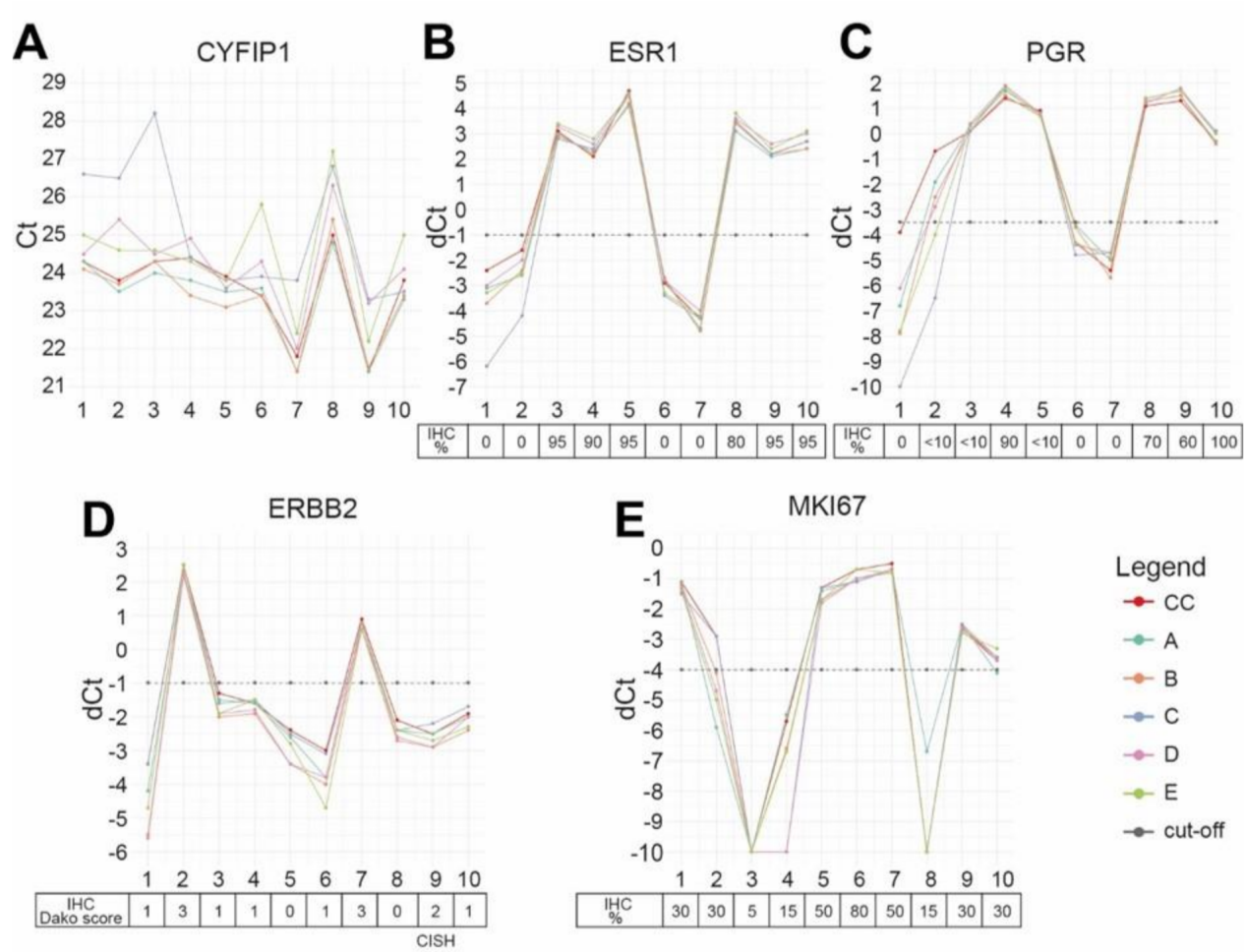

Figure 1. STRAT4 semi-quantitative mRNA level measurements for the reference gene and the four markers of interest. (A) Cytoplasmic FMR1-interacting protein 1 (CYFIP1) cycle threshold (Ct) measurements across the coordination center (CC) and the five participating sites A-E. (B-E) Delta cycle threshold $(\mathrm{dCt})$ measurements for the markers across $\mathrm{CC}$ and the five participating sites A-E: (B) estrogen receptor (ESR1); (C) progesterone receptor (PGR); (D) human epidermal growth factor receptor (ERBB2); (E) Ki-67 (MKI67). Cut-off: dCt cut-off utilized by the STRAT4 assay.

\subsection{Re-Evaluation of Discrepant Cases}

The two cases (case \#2 and case \#10) for which PGR and MKI67 binary results were discrepant were re-evaluated regarding the histopathological features and the immunohistochemical expression of PgR and Ki-67.

Case \#2 presented a pleomorphic invasive lobular breast cancer with heterogeneous low positive $\mathrm{PgR}$ (IHC staining < 10\%), negative ER staining ( $0 \%$ ), HER2-positive staining (IHC 3+), and heterogeneous Ki-67 expression with up to $30 \%$ positive staining on IHC (Figure 2). For this case, two EQA sites had reported discrepant $P G R$ status as negative and 
four sites with negative MKI67 status. IHC was repeated on deeper sections, showing lower PgR expression than in the initial PgR IHC. However, there was still focally very strong expression of PgR (IRS 3/12). The repeated Ki-67 IHC displayed less, but still, expression heterogeneity and confirmed high Ki-67. A repeated STRAT4 analysis of the deeper section delivered the same concordant results as the CC IHC and the first CC STRAT4 testing, with PGR and MKI67 expression values clearly above the dCt cut-off. Yet, it should be noted that PgR IHC showed only low positivity near the cut-off with negative ER staining and Ki-67 a partly heterogeneous but high expression pattern.

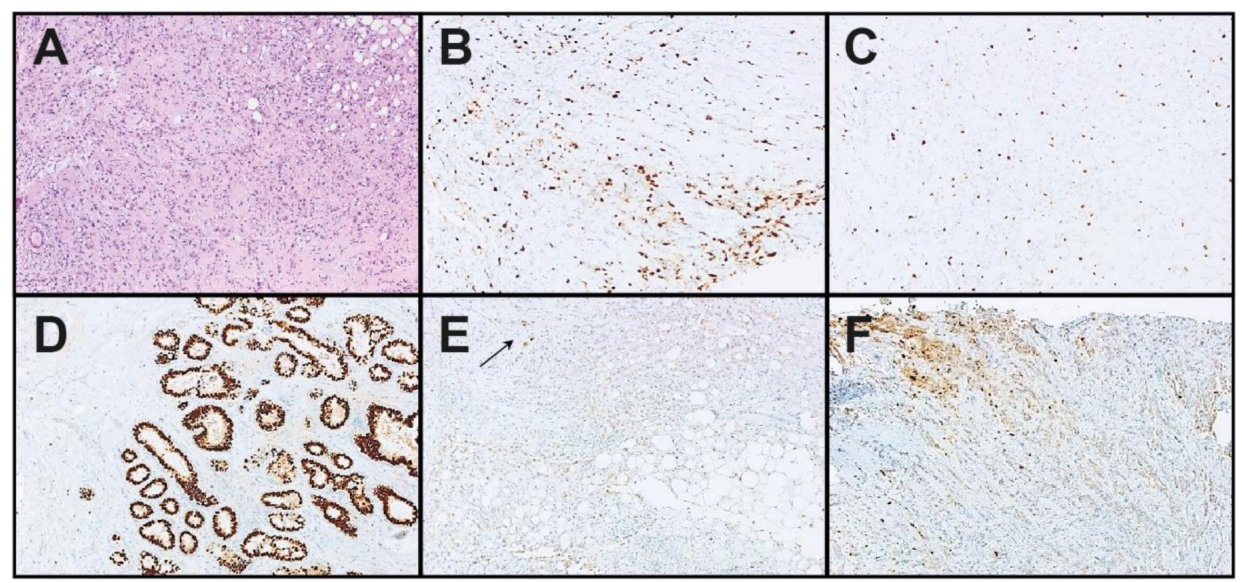

Figure 2. Histological review of one case reported as falsely negative for PGR and MKI67 by two sites. Case \#2 was a pleomorphic invasive lobular breast cancer. (A): Hematoxylin and eosin staining (×200). (B,C): Heterogeneous immunohistochemical (IHC) expression of Ki-67 (×200). (D): Internal positive control (non-neoplastic breast epithelium) in the progesterone receptor $(\mathrm{PgR}) \mathrm{IHC}(\times 200)$. (E,F): Heterogeneous IHC expression of PgR (mostly negative, but focally positive with strong intensity) $(\times 200)$.

Case \#10 was an IBC of no special subtype (NST) that displayed a partly inhomogeneous Ki-67 expression (up to 25-30\%) in both the initial and the second (deeper cut) Ki-67 IHC of the CC. The initial STRAT4 testing in the CC had delivered an MKI67 dCt of -3.7, regarded (given the cut-off of -4.0 ) as positive MKI67 mRNA expression level, which is generally correlated with $>20 \%$ IHC Ki-67 staining, based on the Ki-67 IHC cutoffs used in prior STRAT4 validation studies [26,32]. However, one EQA site had reported MKI67 as negative with a dCt value of -4.1 , just below the cut-off $(-4.0)$. In a second STRAT4 analysis of a deeper section performed by the CC, the reported MKI67 dCt was -4.1, which is slightly below the cut-off. Hence, tumor heterogeneity should be considered in this case, since there were discrepant MKI67 expression values in different adjacent sections of the same tumor tissue.

\section{Discussion}

Here, we presented the first Europe-wide retrospective Xpert ${ }^{\circledR}$ Breast Cancer STRAT4 EQA study. In the study, mRNA-based assessments of ESR1, PGR, ERBB2 and MKI67 expression levels by a cartridge-based, multiplexed test were compared with the diagnostic gold standard, namely immunohistochemical expression of ER, PgR, HER2 (with additional ISH, if necessary), and Ki-67. The aim of our EQA study was to assess the quality and reproducibility of the STRAT4 test in various European institutes that had experience with the test as part of independent clinical studies.

STRAT4 testing was performed on adjacent tissue sections from ten pre-therapy breast core biopsies, containing IBC, by five European pathologic laboratories as well as the coordinating center pathology laboratory. Overall, each participating site passed the EQA criteria. While for ESR1 and ERBB2, results were in perfect agreement with the IHC/ISH subtyping, sensitivity for PGR and MKI67 were, respectively, $94 \%$ and $86 \%$. 
Lower concordance of PGR and MKI67 mRNA levels versus IHC using STRAT4 has been previously described, associated with mRNA/IHC expression levels in an interval close to the cut-offs [26]. In addition, a lower concordance rate for the markers PGR and MKI67 has also been described for another mRNA-based test [33]. Our study thus suggests that, in addition to the binary test result, the absolute $\mathrm{dCt}$ value and its distance to the dCt cut-off should be considered when interpreting the STRAT4 test result for therapeutic stratification.

Lower concordance for PGR and MKI67 was demonstrated in two cases that were reported as falsely negative for both markers by 2 and 4 sites, respectively. The initial IHC stains were reviewed and another IHC for both markers, as well as STRAT4 testing, were repeated on deeper sections. For one case (\#2), both IHC and STRAT4 repeat testing confirmed the initial CC results. However, PgR IHC was only focally strongly expressed (low positive, $<10 \%$ ). Therefore, this expression, very close to the IHC PgR positive cut-off of $\geq 1 \%$, may have hampered PGR mRNA assessment. Repeat Ki-67 expression by IHC in the same case was high $(30 \%)$, but with heterogeneous distribution, which may have caused false negative results. The second discrepant case displayed a partly inhomogeneous, but high $(25-30 \%)$, Ki-67 protein expression on initial and repeat IHC testing. One participating site as well as the repeated STRAT4 test in the CC delivered a negative MKI67 status on repeat IHC testing, which was, however, very close to the cut-off. Most other cases had clear-cut (far from cut-offs) positive or negative expression of ER, PgR, HER2, and Ki-67 in IHC/ISH. Hence, the study showed that mRNA-based testing was very reliable and reproducible in cases that had clear-cut IHC expression, but accuracy might decrease in cases in which expression was close to the predefined cut-offs. However, it should be noted that even for ER, PgR, HER2, and Ki-67 IHC/ISH, definitions of the respective optimal cut-offs are frequently under discussion. For instance, there is no standardized evaluation protocol nor one worldwide-accepted percent staining cut-off for Ki-67 to date [5,6,34], although efforts to harmonize Ki-67 assessment have been recently published [23].

Taken together, Xpert ${ }^{\circledR}$ Breast Cancer STRAT4 mRNA testing for ESR1, PGR, ERBB2, and MKI67 demonstrated good performance when compared to the diagnostic gold standard IHC/ISH. Hence, the mRNA test may be an efficient option for breast cancer subtyping, especially if a laboratory struggles with IHC/ISH evaluation due to pre-analytical, analytical, or post-analytical errors. Pre-analytical issues may be due to inadequate fixatives or time to and of fixation. Another issue may be the size of the tumor area and the low tumor cellularity, respectively, of breast core biopsies. For STRAT4 testing, mRNA automatically extracted from core biopsies is sufficient in most cases (unpublished data of the CC). Analytical errors of IHC/ISH may be caused by inadequate protocols or failure of reagents. Indeed, STRAT4 is a very easy to handle and robust test if implemented according to the manufacturer's instructions. Concerning the reading of IHC/ISH by the pathologist, further problems may occur. First, the assessment may differ between readers causing inter-observer variability. Second, differences in staining procedures and antibody clones may lead to variable staining intensity/percentage. Furthermore, scoring methods, as mentioned above, may differ.

Our EQA study included only ten tumor samples, as is often the case in EQA studies $[35,36]$. It would be interesting to assess the reliability, i.e., the performance relative to reference tests, as well as the predictive and prognostic value of the assay in extensive clinical cohorts. This is currently being performed in a single-center study. Moreover, lacking variability of ER expression and missing an HER2 IHC2+/ISH amplified case are limitations of this study but was due to the fact that we concentrated on different Ki-67 expression levels.

\section{Conclusions}

Our EQA study shows that a cartridge-based mRNA assay might offer a reproducible, standardized and time- and tissue-sparing alternative for the evaluation of ESR1 (ER), PGR (PgR), ERBB2 (HER2), and MKI67 (Ki-67) in IBC. Particularly, it may be an alternative test for consideration if the establishment and implementation of IHC/ISH is hampered due to 
(pre-/post-)analytical or economic reasons. However, tumor heterogeneity and expression patterns by IHC that are close to the defined test cut-offs may deliver false negative or false positive results for cases with borderline reference test results. Hence, reliability, reproducibility, and the assay's prognostic and predictive value regarding pathological complete response should be investigated in large clinical breast cancer cohorts. Currently, we are analyzing this issue in a single-center study.

Author Contributions: Conceptualization, A.H., R.E., J.W., S.B., J.F., I.S.; methodology, A.H., R.E., J.F., J.W., S.B., G.V., F.P.-L., J.P., C.D., T.M.; validation, F.F.; formal analysis, F.F.; investigation, R.E., M.Z., V.P., E.G.R., A.C., T.C.C., P.J., L.K., B.P.-M.; resources, A.H., R.E., H.H., M.R., P.A.F., M.W.B.; data curation, R.E.; writing—original draft preparation, R.E., F.F.; writing—review and editing, all authors; visualization, R.E., F.F.; supervision, A.H., R.S., R.E.; project administration, R.E.; funding acquisition, A.H.; All authors have read and agreed to the published version of the manuscript.

Funding: All Xpert ${ }^{\circledR}$ Breast Cancer STRAT4 (CE-IVD*) kits were provided by Cepheid. Cepheid was not involved in sample selection, nor the final data analysis. ( ${ }^{*} \mathrm{CE}-\mathrm{IVD}$ : In vitro diagnostic medical device. Not available in all countries. Not available in the U.S.)

Institutional Review Board Statement: All procedures performed in studies involving human participants were in accordance with the ethical standards of the local national research committee and with the 1964 Helsinki declaration and its later amendments or comparable ethical standards. Approval for the study was obtained from the local ethics committee at the University of Erlangen [ref. numbers 2700 (approval date: 1 March 2013) and 297_17 Bc (approval date: 11 January 2018)]. This article does not contain any studies with animals performed by any of the authors.

Informed Consent Statement: Informed consent was obtained from all individual participants included in the study.

Data Availability Statement: On request, the data presented in this study are available as summary Excel format from the corresponding authors.

Acknowledgments: We thank our technicians for excellent technical support. Furthermore, we thank all patients for giving their consent to sharing their data with us and for participating in the study.

Conflicts of Interest: A.H. has received honoraria from BMS, MSD, Roche, AstraZeneca, Boehringer Ingelheim, Abbvie, Jansen-Cilag, Diaceutics, Cepheid, Lilly, Agilent, and Ipsen. R.E. has received honoraria from Roche, Eisai, BioNTech, Pfizer, and Novartis. The institution of A.H. and R.E. conducts research for AstraZeneca, Roche, Janssen-Cilag, NanoString Technologies, Novartis, Cepheid, and BioNTech. P.A.F. has received honoraria from Roche, Pfizer, Novartis, and Celgene, and his institution conducts research for Novartis, Cepheid, and BioNTech. F.P-L. has received honoraria or conducts research for AstraZeneca, BMS, MSD, Novartis, Lilly, Roche, Pierre Fabre Oncology, Pfizer, Janssen, and Eisai. C.D. reports personal fees from Teva, Novartis, Pfizer, Roche, Amgen, Celgene, MSD, AstraZeneca, and Daiichi Sankyo and grants from Myriad Genetics, Sividon Diagnostics/Myriad, outside the submitted work. C.D. is a cofounder of Sividon Diagnostics. In addition, C.D. has a patent EP18209672 and software patent (VMscope digital pathology) pending; patent EP20150702464 is issued. E.G.R. has received advisory fees from Thermo Fisher Scientific, Novartis, and MSD Italia and honoraria from Thermo Fisher Scientific, AstraZeneca, and Roche. G.V. has received honoraria from MSD Oncology, Roche, AstraZeneca, Agilent, Daiichi Sankyo, Novartis, and Menarini, and his institution conducts research for Roche, Agilent, and Cepheid. T.M. has received advisory fees or honoraria from BMS, MSD, Roche, AstraZeneca, Boehringer Ingelheim and Lilly. J.W., I.S., and S.B. were all employees of Cepheid at the time of this study. The funders had no role in the selection of samples used in the study, in the collection of data from participating sites, the interpretation of the final data analyses in the study, or the decision to publish the results. The corresponding authors had sole final responsibility for preparing the original draft manuscript, finalizing data analyses, or interpretation of data, in the writing of the manuscript, and in the decision to publish the results. The other authors have no conflicts of interest to declare. 


\section{References}

1. Perou, C.M.; Sorlie, T.; Eisen, M.B.; van de Rijn, M.; Jeffrey, S.S.; Rees, C.A.; Pollack, J.R.; Ross, D.T.; Johnsen, H.; Akslen, L.A.; et al. Molecular portraits of human breast tumours. Nature 2000, 406, 747-752. [CrossRef] [PubMed]

2. Sorlie, T.; Perou, C.M.; Tibshirani, R.; Aas, T.; Geisler, S.; Johnsen, H.; Hastie, T.; Eisen, M.B.; van de Rijn, M.; Jeffrey, S.S.; et al. Gene expression patterns of breast carcinomas distinguish tumor subclasses with clinical implications. Proc. Natl. Acad. Sci. USA 2001, 98, 10869-10874. [CrossRef]

3. Paik, S.; Shak, S.; Tang, G.; Kim, C.; Baker, J.; Cronin, M.; Baehner, F.L.; Walker, M.G.; Watson, D.; Park, T.; et al. A Multigene Assay to Predict Recurrence of Tamoxifen-Treated, Node-Negative Breast Cancer. N. Engl. J. Med. 2004, 351, 2817-2826. [CrossRef]

4. Curigliano, G.; Burstein, H.J.; Winer, E.P.; Gnant, M.; Dubsky, P.; Loibl, S.; Colleoni, M.; Regan, M.M.; Piccart-Gebhart, M.; Senn, H.J.; et al. De-escalating and escalating treatments for early-stage breast cancer: The St. Gallen International Expert Consensus Conference on the Primary Therapy of Early Breast Cancer 2017. Ann. Oncol. 2017, 28, 1700-1712. [CrossRef] [PubMed]

5. Goldhirsch, A.; Winer, E.P.; Coates, A.S.; Gelber, R.D.; Piccart-Gebhart, M.; Thurlimann, B.; Senn, H.J.; Panel, M. Personalizing the treatment of women with early breast cancer: Highlights of the St Gallen International Expert Consensus on the Primary Therapy of Early Breast Cancer 2013. Ann. Oncol. Off. J. Eur. Soc. Med Oncol. 2013, 24, 2206-2223. [CrossRef]

6. Dowsett, M.; Nielsen, T.O.; A’Hern, R.; Bartlett, J.; Coombes, R.C.; Cuzick, J.; Ellis, M.; Henry, N.L.; Hugh, J.C.; Lively, T.; et al. Assessment of Ki67 in breast cancer: Recommendations from the International Ki67 in Breast Cancer working group. J. Natl. Cancer Inst. 2011, 103, 1656-1664. [CrossRef] [PubMed]

7. Gonzalez-Ericsson, P.I.; Stovgaard, E.S.; Sua, L.F.; Reisenbichler, E.; Kos, Z.; Carter, J.M.; Michiels, S.; Le Quesne, J.; Nielsen, T.O.; Lænkholm, A.-V.; et al. The path to a better biomarker: Application of a risk management framework for the implementation of PD-L1 and TILs as immuno-oncology biomarkers into breast cancer clinical trials and daily practice. J. Pathol. 2020, 250, 667-684. [CrossRef]

8. Hammond, M.E.; Hayes, D.F.; Dowsett, M.; Allred, D.C.; Hagerty, K.L.; Badve, S.; Fitzgibbons, P.L.; Francis, G.; Goldstein, N.S.; Hayes, M.; et al. American Society of Clinical Oncology/College of American Pathologists guideline recommendations for immunohistochemical testing of estrogen and progesterone receptors in breast cancer (unabridged version). Arch. Pathol. Lab. Med. 2010, 134, e48-e72. [CrossRef]

9. Allison, K.H.; Hammond, M.E.H.; Dowsett, M.; McKernin, S.E.; Carey, L.A.; Fitzgibbons, P.L.; Hayes, D.F.; Lakhani, S.R.; Chavez-MacGregor, M.; Perlmutter, J.; et al. Estrogen and Progesterone Receptor Testing in Breast Cancer: ASCO/CAP Guideline Update. J. Clin. Oncol. Off. J. Am. Soc. Clin. Oncol. 2020, 38, 1346-1366. [CrossRef]

10. Wolff, A.C.; Hammond, M.E.; Schwartz, J.N.; Hagerty, K.L.; Allred, D.C.; Cote, R.J.; Dowsett, M.; Fitzgibbons, P.L.; Hanna, W.M.; Langer, A.; et al. American Society of Clinical Oncology/College of American Pathologists guideline recommendations for human epidermal growth factor receptor 2 testing in breast cancer. Arch. Pathol. Lab. Med. 2007, 131, 18-43. [CrossRef]

11. Wolff, A.C.; Hammond, M.E.; Hicks, D.G.; Dowsett, M.; McShane, L.M.; Allison, K.H.; Allred, D.C.; Bartlett, J.M.; Bilous, M.; Fitzgibbons, P.; et al. Recommendations for human epidermal growth factor receptor 2 testing in breast cancer: American Society of Clinical Oncology/College of American Pathologists clinical practice guideline update. J. Clin. Oncol. Off. J. Am. Soc. Clin. Oncol. 2013, 31, 3997-4013. [CrossRef] [PubMed]

12. Wolff, A.C.; Hammond, M.E.H.; Allison, K.H.; Harvey, B.E.; Mangu, P.B.; Bartlett, J.M.S.; Bilous, M.; Ellis, I.O.; Fitzgibbons, P.; Hanna, W.; et al. Human Epidermal Growth Factor Receptor 2 Testing in Breast Cancer: American Society of Clinical Oncology/College of American Pathologists Clinical Practice Guideline Focused Update. Arch. Pathol. Lab. Med. 2018, 142, 1364-1382. [CrossRef] [PubMed]

13. Viale, G.; Giobbie-Hurder, A.; Regan, M.M.; Coates, A.S.; Mastropasqua, M.G.; Dell'Orto, P.; Maiorano, E.; MacGrogan, G.; Braye, S.G.; Ohlschlegel, C.; et al. Prognostic and predictive value of centrally reviewed Ki-67 labeling index in postmenopausal women with endocrine-responsive breast cancer: Results from Breast International Group Trial 1-98 comparing adjuvant tamoxifen with letrozole. J. Clin. Oncol. Off. J. Am. Soc. Clin. Oncol. 2008, 26, 5569-5575. [CrossRef] [PubMed]

14. Erber, R.; Gluz, O.; Brunner, N.; Kreipe, H.H.; Pelz, E.; Kates, R.; Bartels, A.; Huober, J.; Mohrmann, S.; Moustafa, Z.; et al. Predictive role of HER2/neu, topoisomerase-II-alpha, and tissue inhibitor of metalloproteinases (TIMP-1) for response to adjuvant taxane-based chemotherapy in patients with intermediate-risk breast cancer: Results from the WSG-AGO EC-Doc trial. Breast Cancer Res. Treat. 2015, 150, 279-288. [CrossRef] [PubMed]

15. Polley, M.Y.; Leung, S.C.; McShane, L.M.; Gao, D.; Hugh, J.C.; Mastropasqua, M.G.; Viale, G.; Zabaglo, L.A.; Penault-Llorca, F.; Bartlett, J.M.; et al. An international Ki67 reproducibility study. J. Natl. Cancer Inst. 2013, 105, 1897-1906. [CrossRef] [PubMed]

16. Hammond, M.E.; Hayes, D.F.; Dowsett, M.; Allred, D.C.; Hagerty, K.L.; Badve, S.; Fitzgibbons, P.L.; Francis, G.; Goldstein, N.S.; Hayes, M.; et al. American Society of Clinical Oncology/College Of American Pathologists guideline recommendations for immunohistochemical testing of estrogen and progesterone receptors in breast cancer. J. Clin. Oncol. Off. J. Am. Soc. Clin. Oncol. 2010, 28, 2784-2795. [CrossRef] [PubMed]

17. Goldhirsch, A.; Wood, W.C.; Gelber, R.D.; Coates, A.S.; Thurlimann, B.; Senn, H.J. Meeting highlights: Updated international expert consensus on the primary therapy of early breast cancer. J. Clin. Oncol. 2003, 21, 3357-3365. [CrossRef]

18. Goldhirsch, A.; Glick, J.H.; Gelber, R.D.; Coates, A.S.; Thurlimann, B.; Senn, H.J. Meeting highlights: International expert consensus on the primary therapy of early breast cancer 2005. Ann. Oncol. Off. J. Eur. Soc. Med Oncol. 2005, 16, 1569-1583. [CrossRef] 
19. Cheang, M.C.U.; Chia, S.K.; Voduc, D.; Gao, D.; Leung, S.; Snider, J.; Watson, M.; Davies, S.; Bernard, P.S.; Parker, J.S.; et al. Ki67 index, HER2 status, and prognosis of patients with luminal B breast cancer. J. Natl. Cancer Inst. 2009, 101, 736-750. [CrossRef]

20. Landmann, A.; Farrugia, D.J.; Zhu, L.; Diego, E.J.; Johnson, R.R.; Soran, A.; Dabbs, D.J.; Clark, B.Z.; Puhalla, S.L.; Jankowitz, R.C.; et al. Low Estrogen Receptor (ER)-Positive Breast Cancer and Neoadjuvant Systemic Chemotherapy: Is Response Similar to Typical ER-Positive or ER-Negative Disease? Am. J. Clin. Pathol. 2018, 150, 34-42. [CrossRef]

21. Chen, T.; Zhang, N.; Moran, M.S.; Su, P.; Haffty, B.G.; Yang, Q. Borderline ER-Positive Primary Breast Cancer Gains No Significant Survival Benefit From Endocrine Therapy: A Systematic Review and Meta-Analysis. Clin. Breast Cancer 2018, 18, 1-8. [CrossRef]

22. AGOEV. Guidelines Breast Version 2020 1D. Available online: https:/ / www.ago-online.de/fileadmin/ago-online/downloads / _leitlinien/kommission_mamma/2020/PDF_DE/2020D\%2004_Pathologie_mit\%20Literatur.pdf (accessed on 21 February 2021).

23. Nielsen, T.O.; Leung, S.C.Y.; Rimm, D.L.; Dodson, A.; Acs, B.; Badve, S.; Denkert, C.; Ellis, M.J.; Fineberg, S.; Flowers, M.; et al. Assessment of Ki67 in Breast Cancer: Updated Recommendations from the International Ki67 in Breast Cancer Working Group. J. Natl. Cancer Inst. 2021, 113, 808-819. [CrossRef] [PubMed]

24. Laible, M.; Schlombs, K.; Kaiser, K.; Veltrup, E.; Herlein, S.; Lakis, S.; Stöhr, R.; Eidt, S.; Hartmann, A.; Wirtz, R.M.; et al. Technical validation of an RT-qPCR in vitro diagnostic test system for the determination of breast cancer molecular subtypes by quantification of ERBB2, ESR1, PGR and MKI67 mRNA levels from formalin-fixed paraffin-embedded breast tumor specimens. BMC Cancer 2016, 16, 398. [CrossRef]

25. Gupta, S.; Mani, N.R.; Carvajal-Hausdorf, D.E.; Bossuyt, V.; Ho, K.; Weidler, J.; Wong, W.; Rhees, B.; Bates, M.; Rimm, D.L. Macrodissection prior to closed system RT-qPCR is not necessary for estrogen receptor and HER2 concordance with IHC/FISH in breast cancer. Lab. Investig. 2018, 98, 1076-1083. [CrossRef] [PubMed]

26. Wu, N.C.; Wong, W.; Ho, K.E.; Chu, V.C.; Rizo, A.; Davenport, S.; Kelly, D.; Makar, R.; Jassem, J.; Duchnowska, R.; et al. Comparison of central laboratory assessments of ER, PR, HER2, and Ki67 by IHC/FISH and the corresponding mRNAs (ESR1, PGR, ERBB2, and MKi67) by RT-qPCR on an automated, broadly deployed diagnostic platform. Breast Cancer Res. Treat. 2018, 172, 327-338. [CrossRef] [PubMed]

27. Filipits, M.; Rudas, M.; Singer, C.; Bago-Horvath, Z.; Greil, R.; Balic, M.; Lax, S.; Wu, N.; Zhao, S.; Weidler, J.; et al. mRNA Expression of ER, PR, HER2 and Ki67 are Concordant with Central IHC and Predict Clinical Outcome: A Validation Study from the ABCSG-6 Biomarker Cohort. Ann. Oncol. 2018, 29 (Suppl. 8), 1339. [CrossRef]

28. Remmele, W.; Stegner, H.E. Recommendation for uniform definition of an immunoreactive score (IRS) for immunohistochemical estrogen receptor detection (ER-ICA) in breast cancer tissue. Pathologe 1987, 8, 138-140. [PubMed]

29. Bethune, G.C.; Veldhuijzen van Zanten, D.; MacIntosh, R.F.; Rayson, D.; Younis, T.; Thompson, K.; Barnes, P.J. Impact of the 2013 American Society of Clinical Oncology/College of American Pathologists guideline recommendations for human epidermal growth factor receptor 2 (HER2) testing of invasive breast carcinoma: A focus on tumours assessed as 'equivocal' for HER2 gene amplification by fluorescence in-situ hybridization. Histopathology 2015, 67, 880-887. [CrossRef] [PubMed]

30. Viale, G.; On the behalf of the International Breast Cancer Study Group; Regan, M.M.; Mastropasqua, M.G.; Maffini, F.; Maiorano, E. Predictive Value of Tumor Ki-67 Expression in Two Randomized Trials of Adjuvant Chemoendocrine Therapy for NodeNegative Breast Cancer. JNCI J. Natl. Cancer Inst. 2008, 100, 207-212. [CrossRef]

31. R Core Team. R: A Language and Environment for Statistical Computing. R Foundation for Statistical Computing: Vienna, Austria, 2019. Available online: https:/ / www.R-project.org/ (accessed on 21 February 2021).

32. Xpert Breast Cancer STRAT4 (CE-IVD) package insert. 301-4981, Rev. C March 2020.

33. Wirtz, R.M.; Sihto, H.; Isola, J.; Heikkilä, P.; Kellokumpu-Lehtinen, P.L.; Auvinen, P.; Turpeenniemi-Hujanen, T.; Jyrkkiö, S.; Lakis, S.; Schlombs, K.; et al. Biological subtyping of early breast cancer: A study comparing RT-qPCR with immunohistochemistry. Breast Cancer Res. Treat. 2016, 157, 437-446. [CrossRef] [PubMed]

34. Harris, L.; Fritsche, H.; Mennel, R.; Norton, L.; Ravdin, P.; Taube, S.; Somerfield, M.R.; Hayes, D.F.; Bast, R.C., Jr. American Society of Clinical Oncology 2007 update of recommendations for the use of tumor markers in breast cancer. J. Clin. Oncol. Off. J. Am. Soc. Clin. Oncol. 2007, 25, 5287-5312. [CrossRef] [PubMed]

35. Kirchner, M.; Glade, J.; Lehmann, U.; Merkelbach-Bruse, S.; Hummel, M.; Lehmann, A.; Trautmann, M.; Kumbrink, J.; Jung, A.; Dietmaier, W.; et al. NTRK testing: First results of the QuiP-EQA scheme and a comprehensive map of NTRK fusion variants and their diagnostic coverage by targeted RNA-based NGS assays. Genes Chromosomes Cancer 2020, 59, 445-453. [CrossRef]

36. Jöhrens, K.; von Wasielewski, R.; Kreipe, H.H.; Forberger, A.; Jurmeister, P.; Dietel, M.; Stenzinger, A.; Fischer, J. Quality assurance in diagnostic in situ hybridization-experience of QuIP. Pathologe 2020, 41, 614-620. [CrossRef] 